\title{
Previous psychopathology predicted severe COVID-19 concern, anxiety, and PTSD symptoms in pregnant women during "lockdown" in Italy
}

\author{
Claudia Ravaldi ${ }^{1,2,3}$ - Valdo Ricca ${ }^{4}$. Alyce Wilson ${ }^{5}$ - Caroline Homer ${ }^{5}$ - Alfredo Vannacci ${ }^{1,2,3}$ (D)
}

Received: 30 July 2020 / Accepted: 31 October 2020 / Published online: 20 November 2020

(C) Springer-Verlag GmbH Austria, part of Springer Nature 2020

\begin{abstract}
Italy was the first COVID-19 pandemic epicenter among European countries and established a period of full "lockdown", consisting of travel bans, mandatory staying at home, and temporary closure of nonessential businesses. Similar measures are known risk factors for psychological disturbances in the general population; still, little is known about their impact on pregnant women's mental health during COVID-19 pandemic. The cross-sectional, web-based, national survey "COVID-19 related Anxiety and StreSs in prEgnancy, poSt-partum and breaStfeeding" (COVID-ASSESS) was conducted during the first month of full "lockdown" in Italy. Participants were recruited via social networks with a snowball technique. The questionnaire was specifically developed to examine COVID-19 concerns and included the psychometric tests National Stressful Events Survey (NSESSS) for posttraumatic stress disorder (PTSD) and State-Trait Anxiety Inventory. A multivariable logistic regression model was fitted to explore the association of the concern, anxiety and PTSD symptoms with age, gestational weeks, parity, days of "lockdown", assisted reproductive technology use, psychopathological history, and previous perinatal losses. Out of 1015 pregnant women reached, 737 (72.6\%) fully answered the questionnaire; no woman reported a COVID-19 infection. Median age was 34.4 years [quartiles 31.7,37.2], median days in "lockdown" were 13.1 [11.0, 17.0], median gestational weeks were 27.8 $[19.8,34.0]$. Clinically significant PTSD symptoms were present in 75 women (10.2\%, NSESSS cutoff 24) and clinically significant anxiety symptoms were present in 160 women (21.7\%, STAI-Y1 cutoff 50). Women were particularly worried about the health of their baby and of their elderly relatives, as well as of the possible impact of pandemic in the future of society. Previous anxiety predicted higher concern and PTSD symptoms; previous depression and anxiety were independently associated with current PTSD symptoms.
\end{abstract}

Keywords COVID-19 $\cdot$ Pregnancy $\cdot$ PTSD $\cdot$ Anxiety

This article is part of the Topical Collection on Women's mental health during the Covid-19 pandemic

Edited by: Anita Riecher-Rössler

Alfredo Vannacci

alfredo.vannacci@unifi.it

Claudia Ravaldi

claudia.ravaldi@unifi.it

Valdo Ricca

valdo.ricca@unifi.it

Alyce Wilson

alyce.wilson@burnet.edu.au

Caroline Homer

caroline.homer@burnet.edu.au
1 PeaRL Perinatal Research Laboratory, University of Florence, Prato, Italy

2 CiaoLapo Foundation for Perinatal Health, Prato, Italy

3 Department of Neurosciences, Psychology, Drug Research and Child Health, University of Florence, viale Pieraccini 6, 50139 Florence, Italy

4 Pychiatry Unit, Department of Health Sciences, University of Florence, Florence, Italy

5 Maternal, Child and Adolescent Health Program, Burnet Institute, Melbourne, Victoria, Australia 


\section{Background}

The newly identified Coronavirus SARS-CoV-2, responsible for the associated respiratory infection designated COVID-19, was first identified in December 2019 in Wuhan, China, and spread to Europe and worldwide within months. Italy was the first pandemic epicenter among European countries. On 9 March 2020, the Italian Government established a period of full "lockdown", consisting of travel bans, mandatory staying at home for all (except for emergencies, health problems or regulated shopping for bare necessities) and temporary closure of nonessential shops and businesses, that lasted until 3 May 2020. These measures, initially considered strict and controversial, eventually controlled SARS-CoV-2 transmission in all Italian regions. In the following months, similar measures have been implemented in many European (Lavezzo et al. 2020) and other countries globally to control the pandemic. Quarantine and selfisolation are now common practices in many countries to prevent transmission of the virus. In nonpandemic times, quarantine and social isolation are a well-known risk factors for psychological and psychiatric disturbances in the general population (Brooks et al. 2020; Usher et al. 2020), particularly for children and adolescents, the elderly, those from lower socioeconomic groups, females, and people with preexisting mental health conditions (Perrin et al. 2009). However, little is known about the impact on the mental health of pregnant women during the COVID-19 pandemic.

\section{Methods}

During the first period of full "lockdown" in Italy (18-31 March 2020), we conducted a national survey "COVID-19 related Anxiety and StreSs in prEgnancy, poSt-partum and breaStfeeding" (COVID-ASSESS) to investigate the psychological impact of the pandemic and 'lockdown' on pregnant women. The research methods are described elsewhere (Ravaldi et al. 2020; Ravaldi and Vannacci 2020). Briefly, a cross-sectional, web-based study was conducted using an online questionnaire. Participants were recruited via social networks with a snowball technique and sponsored social network advertisements. Approval for the study was obtained from the ethics committee of Florence University (Università degli Studi di Firenze, Commissione per l'Etica della Ricerca di Ateneo). Participants self-selected to complete the survey, participation was voluntary and all participants gave their consent in an online form. The online survey-known as the COVID-ASSESS questionnaire - comprised of a sociodemographic section, a specifically developed survey to examine concerns related to the COVID-19 pandemic (rated on a Likert scale 0-3 from "not at all concerned" to "very concerned") and two validated psychometric tests: the National Stressful Events Survey (NSESSS) for posttraumatic stress disorder (PTSD) (LeBeau et al. 2014) and the State-Trait Anxiety Inventory form $Y$ (STAI-Y) (Spielberger et al. 1983).

Categorical variables were reported as $n(\%)$, continuous variables as median [quartiles; range min-max]; A multivariable logistic regression model was fitted to explore the association of the selected outcomes (concern, anxiety and PTSD symptoms) with age, gestational weeks, parity, days of "lockdown", assisted reproductive technology use, self-reported psychopathological history, and previous perinatal losses (for the purpose of this study, stillbirth and neonatal infant death were grouped together, since they exert similar psychopathological impact on bereaved parents). Analyses were conducted with Stata/IC 16.1 (StataCorp), a value of $p<0.01$ was considered statistically significant. Graphs were plotted with Tableau Desktop 2020.1 (Tableau Software, LLC).

\section{Results}

Although response rates could not be exactly quantifiable due to the self-selected and nonprobabilistic nature of the sample (snowball technique), 737 out of 1015 pregnant women who logged in to the web-based survey, fully answered the COVID-ASSESS questionnaire (72.6\%); no woman reporting having experienced a COVID-19 infection. The median age was 34.4 years [31.7, 37.2; range 18.4-47.4], median days in "lockdown" at the time of interview were 13.1 [11.0, 17.0; range 7.8-27.5]; 263 women $(35.7 \%)$ were at their first pregnancy (no previous childbirth, no previous pregnancy loss), median gestational weeks were 27.8 [19.8, 34.0; range 4.742.5], and the distribution of pregnancy trimesters was first trimester 67 (9.1\%), second trimester 309 (41.9\%), and third trimester $361(49.0 \%)$. Assisted reproductive technology was reported by $45(5.8 \%)$ women and 277 women $(37.6 \%)$ reported at least one previous pregnancy or postnatal loss [miscarriages 204 (27.7\%); termination of pregnancy 75 (10.2\%); stillbirths or neonatal/infant losses 36 (4.8\%)].

Previous psychopathological diagnoses were self-reported by 293 women (39.8\%), in particular: anxiety 241 (32.7\%), depression $69(9.3 \%)$, bipolar disorder $4(0.5 \%)$, obsessive compulsive disorder (OCD) 9 (1.2\%), and eating disorders 46 (6.2\%). Women with a history of anxiety were significantly more concerned about COVID-19. On a Likert scale from 0 to 3, women with no previous psychopathological diagnosis scored a median of 2.28 [2.00; 2.71$]$, vs 2.43 [2.14; 2.71$]$ of women with a psychopathological history ( $p=0.003$ ); the difference was more pronounced regarding health-related issues (Fig. 1a, blue bars), than society-related issues (Fig. 1a, gray bars). Consistently, a previous diagnosis of anxiety was the only factor able to significantly predict current high levels of concern during "lockdown" period, in the multivariate analysis (OR 1.85; CI 1.16, 2.95).

With regard to psychopathological outcomes, clinically significant PTSD symptoms were present in 75 women 
a

\section{COVID-19 concerns}
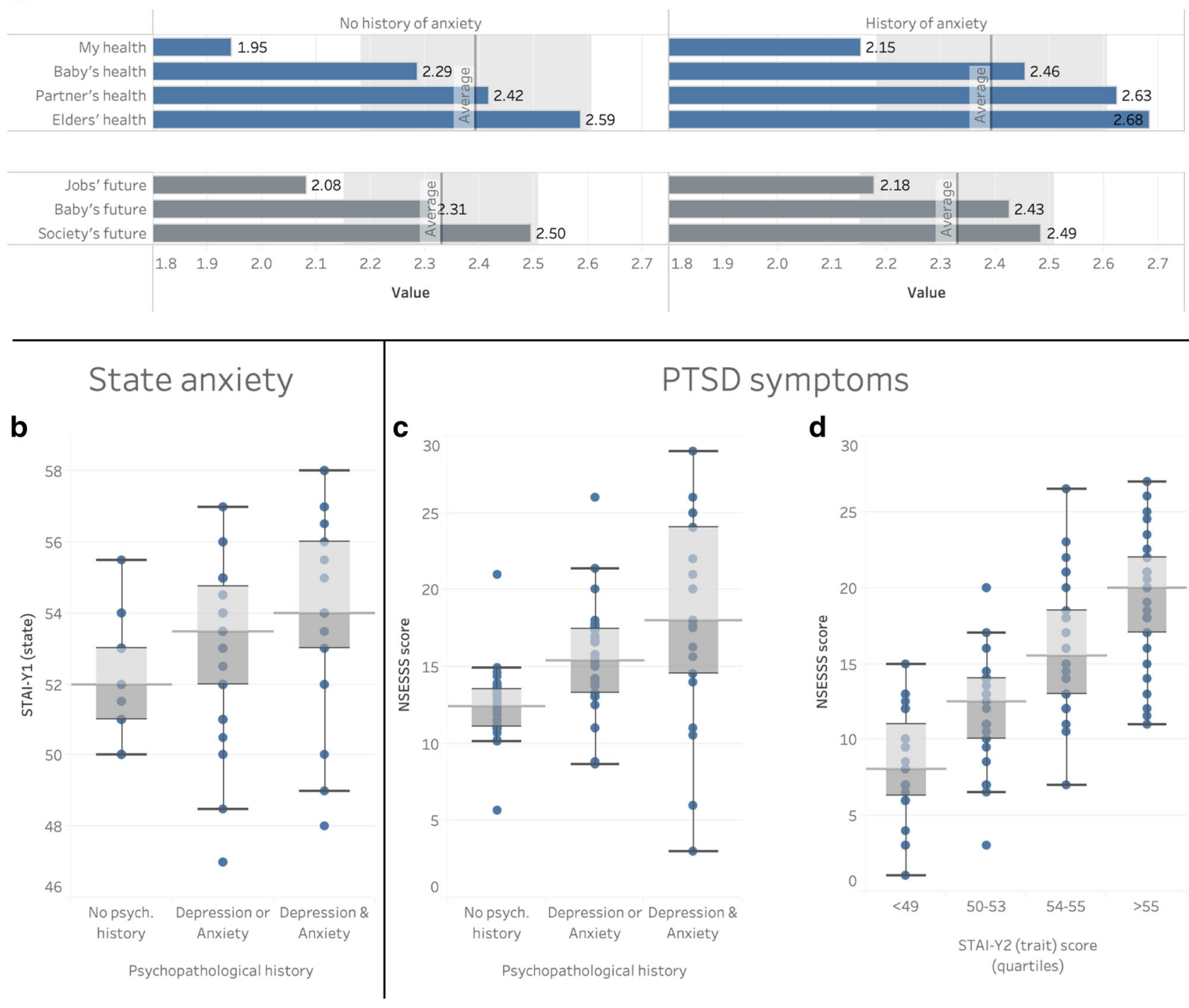

symptoms

d

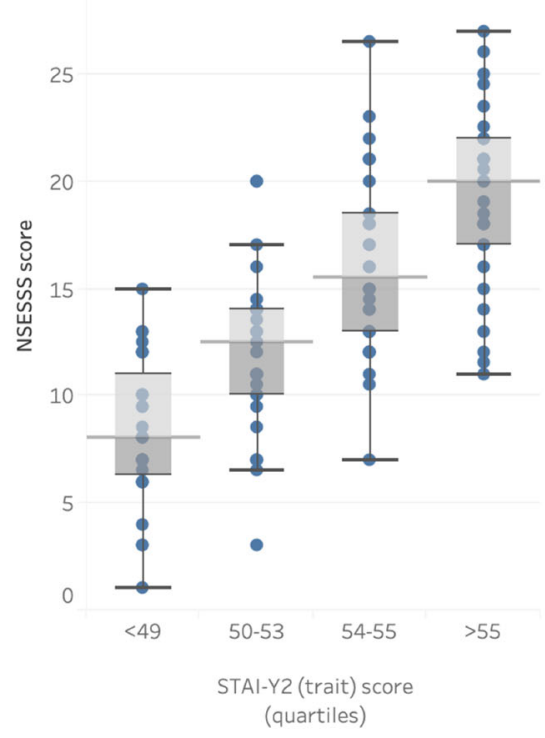

Fig. 1 Level of concern of women according to history of psychological distress (a); state anxiety according to psychopathological history (b); posttraumatic stress disorder (PTSD) symptoms according to psychopathological history (c) and anxiety trait (d)

(10.2\%) using a NSESSS cutoff of 24 (Cimino et al. 2019), and clinically significant anxiety symptoms were present in 160 women (21.7\%) using a STAI-Y1 cutoff of 50 (Tersigni et al. 2018). The most important factors correlated to high levels of psychopathology were previous diagnoses of anxiety and/or depression. Women with a history of anxiety or depression showed significantly higher levels of anxiety, measured as STAI-Y1 score (Fig. 1b; no previous psychopathology 52 $[49 ; 55]$ vs previous depression or anxiety $53[51 ; 56]$ vs previous depression and anxiety $54[51 ; 56] ; p=0.0001$ ).

Finally, women with a history of anxiety or depression showed significantly more PTSD symptoms (Fig. 1c; no previous psychopathology 12 [7;17], previous depression or anxiety 16 [10;22], previous depression and anxiety 19 [11; $25] ; p=0.0001)$. Previous depression and anxiety diagnoses were also independently associated with the risk of developing PTSD symptoms in the multivariate analysis $(p=0.004)$ : previous depression OR $2.31(1.18,4.50)$, previous anxiety OR $2.30(1.38,3.82)$, and previous depression and anxiety OR $5.66(2.56,12.54)$. Consistently, women with an anxiety trait (measured by STAI-Y2) showed higher levels of PTSD symptoms (Fig. 1d; Rsq $=0.11, p=0.0001$ ).

Results of this nationwide survey conducted during the first period of "lockdown" in Italy show that SARS-CoV-2-negative pregnant women were very concerned about COVID-19 and showed a high prevalence of anxiety and posttraumatic stress disorder symptoms. Higher levels of concern and psychological distress were significantly associated with a previous psychopathological diagnosis. In particular, women with self-reported history of anxiety and/or depression were 
significantly more concerned about COVID-19 and were at a higher risk of developing symptoms of anxiety and posttraumatic stress disorder.

These findings have immediate relevance for healthcare professionals caring for pregnant and postnatal women during the COVID-19 pandemic. When taking a medical history, it is critical that care givers ask women about any history of anxiety and/or depression as this seems to be the most important factor in predicting COVID-19 associated distress and psychopathology during pregnancy. Healthcare professionals caring for pregnant and postnatal women during current or future public health crises should be aware that the simple act of asking women about previous psychopathological diagnoses (although only self-reported) can be an easy and useful way to identify patients in need of particular care and attention, in order to reduce the risk of adverse psychological outcomes, such as PTSD and postpartum depression.

Acknowledgment CiaoLapo Foundation for Healthy Pregnancy and Perinatal Loss Support provided infrastructure for the realization of the study (documents, questionnaires, material, software, web platforms, open access etc.).

\section{Compliance with ethical standards}

Conflict of interest The authors declare that they have no conflict of interest.

\section{References}

Brooks SK, Webster RK, Smith LE, Woodland L, Wessely S, Greenberg N, Rubin GJ (2020) Rapid review the psychological impact of quarantine and how to reduce it: rapid review of the evidence. Lancet 395:912-920. https://doi.org/10.1016/S0140-6736(20) 30460-8

Cimino AN, Yi G, Patch M, Alter Y, Campbell JC, Gundersen KK, Tang JT, Tsuyuki K, Stockman JK (2019) The effect of intimate partner violence and probable traumatic brain injury on mental health outcomes for black women. J Aggress Maltreat Trauma 28:714-731. https://doi.org/10.1080/10926771.2019.1587657

Lavezzo E, Franchin E, Ciavarella C et al (2020) Suppression of a SARSCoV-2 outbreak in the Italian municipality of Vo'. Nature. 584:425429. https://doi.org/10.1038/s41586-020-2488-1

LeBeau R, Mischel E, Resnick H, Kilpatrick D, Friedman M, Craske M (2014) Dimensional assessment of posttraumatic stress disorder in DSM-5. Psychiatry Res 218:143-147. https://doi.org/10.1016/j. psychres.2014.03.032

Perrin PC, McCabe OL, Everly GS, Links JM (2009) Preparing for an influenza pandemic: mental health considerations. Prehosp Disaster Med 24:223-230. https://doi.org/10.1017/S1049023X00006853

Ravaldi C, Vannacci A (2020) The COVID-ASSESS dataset - COVID19 related anxiety and stress in prEgnancy, poSt-partum and breaStfeeding during lockdown in Italy. Data in Brief. 33: 106440. https://doi.org/10.1016/j.dib.2020.106440

Ravaldi C, Wilson A, Ricca V, Homer C, Vannacci A (2020) Pregnant women voice their concerns and birth expectations during the COVID-19 pandemic in Italy. Women Birth. https://doi.org/10. 1016/j.wombi.2020.07.002

Spielberger C, Gorssuch R, Lushene P et al (1983) Manual for the StateTrait Anxiety Inventory STAI (form Y). Consulting Psychologists Press, Palo Alto

Tersigni C, D'Ippolito S, Di Nicuolo F et al (2018) Recurrent pregnancy loss is associated to leaky gut: a novel pathogenic model of endometrium inflammation? J Transl Med 16:102. https://doi.org/10. 1186/s12967-018-1482-y

Usher K, Bhullar N, Jackson D (2020) Life in the pandemic: social isolation and mental health. J Clin Nurs 29:2756-2757. https://doi.org/ 10.1111 jocn. 15290

Publisher's note Springer Nature remains neutral with regard to jurisdictional claims in published maps and institutional affiliations. 